\title{
Regulatory effect of Bcl-2 in ultraviolet radiation-induced apoptosis of the mouse crystalline lens
}

\author{
YUCHEN DONG, YAJUAN ZHENG, JUN XIAO, CHAO ZHU and MEISHENG ZHAO \\ Department of Ophthalmology, Eye Hospital of The Second Clinical Hospital of Jilin University, \\ Jilin University, Changchun, Jilin 130051, P.R. China
}

Received March 7, 2014; Accepted September 10, 2014

DOI: 10.3892/etm.2015.2960

\begin{abstract}
The aim of the present study was to analyze the role of Bcl-2 during the process of apoptosis in the mouse crystalline lens. In total, 12 normal mice served as the control group and 12 Bcl-2 knockout (K.O) mice served as the experimental group. The mouse crystalline lens was sampled for the detection of Bcl-2 and caspase-3 expression following exposure to ultraviolet (UV) radiation. Reverse transcription-quantitative polymerase chain reaction (RT-qPCR) was used to determine $\mathrm{Bcl}-2$ expression in the groups of normal mice receiving $\mathrm{UV}$ radiation or not receiving UV radiation. Samples of the murine crystalline lens were microscopically harvested and analyzed using western blotting. Apoptosis was detected using terminal deoxynucleotidyl transferase dUTP nick-end labeling (TUNEL) assay. Furthermore, caspase 3 activity was examined using enzyme-linked immunosorbent assay kits, and RT-qPCR was used to analyze caspase-3 expression levels. The results of the present study demonstrated that there was no statistically significant difference in the level of Bcl-2 gene transcription between the two groups. In addition, UV radiation did not change the macrostructure of the crystalline lens in the group of normal mice or the group of Bcl-2 K.O mice. The results of the TUNEL assay indicated that the normal-UV group exhibited a more significant apoptosis level compared with the Bcl-2 K.O-UV group. Furthermore, the mRNA expression level of caspase-3 in the normal-UV group was significantly higher compared with the normal-nonUV group $(\mathrm{P}<0.05)$, while the levels in the Bcl-2 K.O-UV group were significantly higher compared with the Bcl-2 K.O and normal-nonUV groups $(\mathrm{P}<0.05)$. In addition, the mRNA expression level of caspase-3 was significantly higher in the normal-UV, as compared with the Bcl-2 K.O-UV group $(\mathrm{P}<0.05)$, and the variation trends in caspase-3 activity were consistent. In conclusion, the results of the present study demonstrated that Bcl-2 may have an
\end{abstract}

Correspondence to: Dr Meisheng Zhao, Department of Ophthalmology, Eye Hospital of the Second Clinical Hospital of Jilin University, Jilin University, 218 Ziqiang Street, Changchun, Jilin 130051, P.R. China

E-mail: zhaomeishengprof@yeah.net

Key words: Bcl-2, ultraviolet, crystalline lens, apoptosis important role in the promotion of $\mathrm{UV}$-induced apoptosis in the crystalline lens.

\section{Introduction}

Ultraviolet (UV) radiation and oxidative stress may lead to the formation of cataracts; thus, a number of appropriate mechanisms and relevant theories have been proposed to explain the formation of lens opacities $(1,2)$. Eyes have a certain capacity to resist damage; however, for cataract patients, functional defects in the defense against UV and oxidative stress may enable damage factors to cause epithelial apoptosis in the crystalline lens more easily, which may be the cytological basis underlying the formation of cataracts (3). Generally, UV exposure may lead to a series of complex acute or chronic reactions and produce a surplus reactive oxygen species, including hydrogen peroxide and peroxide ions $(4,5)$. Degradation, cross-linking and polymerization of proteins in the crystalline lens may occur if the reactive oxygen species are not eliminated by reduction and accumulate substantially (6). In addition, UV radiation may lead to DNA damage, including the formation of base-free loci and pyrimidine dimers, which severely affect DNA duplication and transcription. Subsequently, DNA damage may be repaired to enable the cells to survive continuously, while programed cell death or apoptosis may be utilized to eliminate the defective individual cells; thus, maintaining the structural and functional completeness of the crystalline lens $(7,8)$. However, the intrinsic physiological functions of the crystalline lens are likely to be affected, resulting in a series of pathological changes if apoptosis occurs to a number of cells, or if the apoptotic cells can not be offset by proliferation $(9,10)$. UV radiation is an inevitable environmental factor in daily lives and is closely associated with the onset of ophthalmic diseases. Thus, research on UV-induced apoptosis in the crystalline lens should provide more understanding into the pathogenesis of cataracts.

\section{Materials and methods}

Materials. $\mathrm{A} \mathrm{CO}_{2}$ incubator was purchased from Shanghai Aoxin Technology Instrument Co., Ltd. (Shanghai, China); the chemical luminous gel-imaging system was purchased from Hach Company (Loveland, CO, USA); and the cryogenic desk centrifuge was purchased from Beijing Jingli Centrifuge Co., Ltd. (Beijing, China). 
Laboratory Bcl-2 knockout (K.O) mice and normal control mice were provided by Shanghai SLAC Laboratory Animal Co., Ltd. (Shanghai, China). The present study was approved by the Ethics Committee of The Second Hospital of Jilin University (Changchun, China) and was performed according to the Care and Use of Laboratory Animals Guidelines of China.

Grouping. In total, 12 normal control mice served as the control group and $12 \mathrm{Bcl}-2 \mathrm{~K} . \mathrm{O}$ mice served as the experimental group. The mice were aged $\sim 12$ weeks in the two groups. One eye of each mouse was selected randomly for UV irradiation, while the other eye was covered and used as the control. Thus, the mice were further divided into four groups, which included the normal-nonUV, normal-UV, Bcl-2 K.O-nonUV and Bcl-2 K.O-UV groups.

Irradiation of the crystalline lens by $U V$. In the mice, one eye was exposed to the UV radiator (SP3-250D; Ushio Electric Co., Tokyo, Japan), while the other eye was covered with a lead sheet patch. The total UV radiation dosage was $8 \mathrm{~kJ} / \mathrm{m}^{2}$ and the radiation time was $15 \mathrm{~min}$. UV radiation was produced by the UV radiator. The radiation level was determined using an energy meter (GTFP200A7J; Meba Electric Co., Ltd., CA, USA) at the cornea plane, and the UV spectrum was recorded using a spectrometer (F4600; Hitachi, Ltd., Tokyo, Japan). The UV spectra ranged between 280 and $320 \mathrm{~nm}$.

Test sample collection and observation of the crystalline lens macrostructure. Mice were anesthetized with diethyl ether and sacrificed by cervical fracture following irradiation with UV for $24 \mathrm{~h}$. The two eyeballs were enucleated (optic nerve with an approximate length of $2 \mathrm{~mm}$ was retained). Samples of the mouse crystalline lens were microscopically obtained for reverse transcription-quantitative polymerase chain reaction (RT-qPCR) and caspase-3 activity determination. The samples were also weighed. The crystalline lens was washed three times with diethylpyrocarbonate-phosphate-buffered saline (PBS) for use in subsequent experiments.

Tissue fixation and preparation of the paraffin sections. Glacial acetic acid solution, formaldehyde solution, normal saline solution and $75 \%$ ethanol solution were mixed in the ratio 1:2:7:10 to form a fixation solution. The entire eyeball was immersed in the fixation mixture and fixed for $0.5 \mathrm{~h}$ at room temperature. Once the eyeball surface had become hard, the eyeballs were injected with the fixation solution from the posterior chamber and fixed for $2 \mathrm{~h}$. Samples were incised from the upper and lower sections using a sharp blade or ophthalmological scissors, and subsequently fixed for $2 \mathrm{~h}$ at room temperature. The samples were then immersed in $10 \%$ neutral formaldehyde and fixed for a further 20 h. Following a series of dehydrations using gradient concentrations of ethanol, the samples were blocked with dimethylbenzene solution, embedded in paraffin at $60^{\circ} \mathrm{C}$ and cut into $4 \mu \mathrm{m}$ sections.

Terminal deoxynucleotidyl transferase dUTP nick-end labeling (TUNEL) assay. Following drop-wise addition of $50 \mu 1$ alkali Triton X-100 (0.1\%; prepared by $0.1 \%$ sodium citrate), the samples were incubated for $8 \mathrm{~min}$ at room temperature, and washed twice with PBS for 5 min. Next, $50 \mu 1 \mathrm{H}_{2} \mathrm{O}_{2}(3 \%)$ was added in a drop-wise manner and the samples were left for $10 \mathrm{~min}$ at room temperature, followed by two washes with PBS for $5 \mathrm{~min}$. Following drop-wise addition of $50 \mu 1$ TUNEL reaction solution, the samples were incubated for $2 \mathrm{~h}$ at $37^{\circ} \mathrm{C}$ in the dark, and washed twice with PBS for $5 \mathrm{~min}$. The staining results were observed microscopically following subsequent procedures, as described in the relevant literature (11).

Determination of caspase-3 activity. An appropriate volume of radioimmunoprecipitation assay lysis buffer was added according to the mass and volume of each specimen. The samples were placed on ice, pipetted into a single-cell suspension, and subsequently placed on ice for a further $5 \mathrm{~min}$. The samples were centrifuged for $10 \mathrm{~min}$ at $10,000 \mathrm{x} \mathrm{g}$ at $4^{\circ} \mathrm{C}$. Protein extract was obtained by separating the supernatant and a caspase-3 detection kit was used to determine the activity of caspase-3, as described in a previous study (12).

Statistical analysis. SPSS 12.0 statistical analysis software (SPSS, Inc., Chicago, IL, USA) was used for the statistical analyses, and the data are expressed as the mean \pm standard deviation. An independent t-test was conducted for the comparison of mean values between samples, where $\mathrm{P}<0.05$ was considered to indicate a statistically significant difference.

\section{Results}

Expression of Bcl-2 in the crystalline lens of the normal mice. Total RNA was extracted from the crystalline lens of mice by using the Total RNA Extraction kit (Tiangen Biotech Co., Ltd., Beijing, China). Total RNA $(0.2 \mu \mathrm{g})$ was subjected to RT-qPCR and the amplification products were subjected to agarose gel electrophoresis. The results demonstrated that the mRNA expression level of Bcl-2 was similar to that of the control gene, $\beta$-actin, in the normal-UV and normal-nonUV groups. Thus, a considerable level of Bcl-2 transcription was observed in the crystalline lens of the adult mice (Fig. 1).

$U V$ radiation does not affect Bcl-2 expression. A statistically significant difference was not observed in the expression levels of Bcl-2 between the normal-UV and normal-nonUV groups (Fig. 1), indicating that the UV irradiation did not trigger Bcl-2 mRNA transcription. In addition, UV radiation did not induce any changes in the mRNA expression of Bcl-2 in the Bcl-2 K.O groups (Fig. 1).

No significant changes occur to the macrostructure of the crystalline lens. UV radiation did not cause lens opacities in the various specimens of the normal and Bcl-2 K.O mice. Furthermore, no characteristic changes of cell apoptosis, including hydrofracturing and vacuoles, were detected. Thus, the results indicated that the UV irradiation dose used in the experiment was insufficient to cause lens opacities prior to the sacrifice of the mice.

$U V$ radiation induces apoptosis in the crystalline lens. A TUNEL assay kit was used to investigate the rate of apop- 


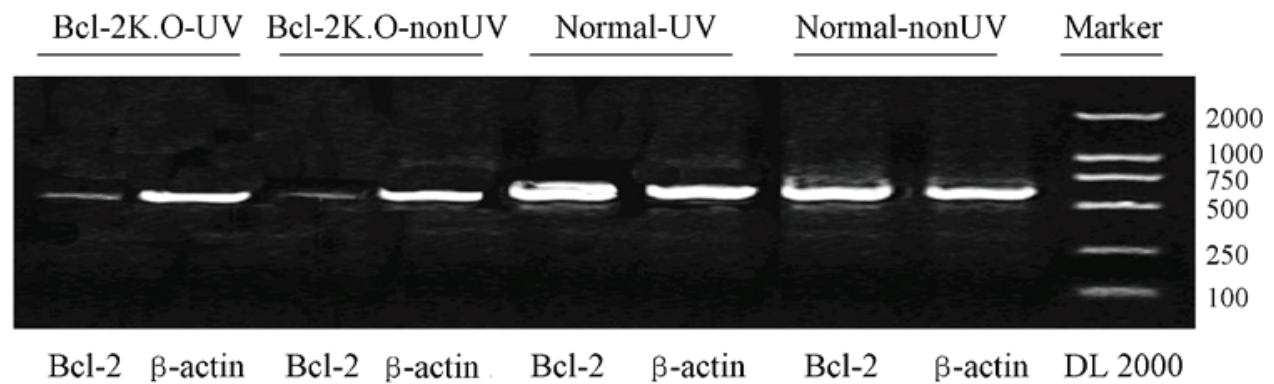

Figure 1. Effect of UV radiation on Bcl-2 in the crystalline lens of normal mice and Bcl-2 gene K.O mice. Bcl-2 gene transcription, as indicated by agarose gel electrophoresis following reverse transcription-quantitative polymerase chain reaction amplification in each group. Bcl-2 gene expression was observed in the cells of the crystalline lens in the normal mice with or without UV treatment. UV, ultraviolet; K.O, knockout. $\beta$-actin was used as an internal reference.

A

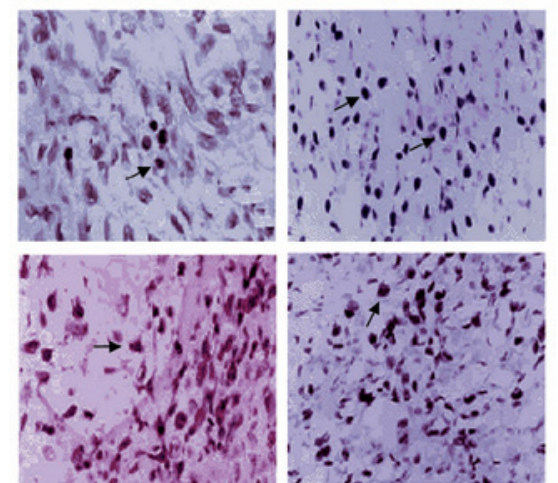

B

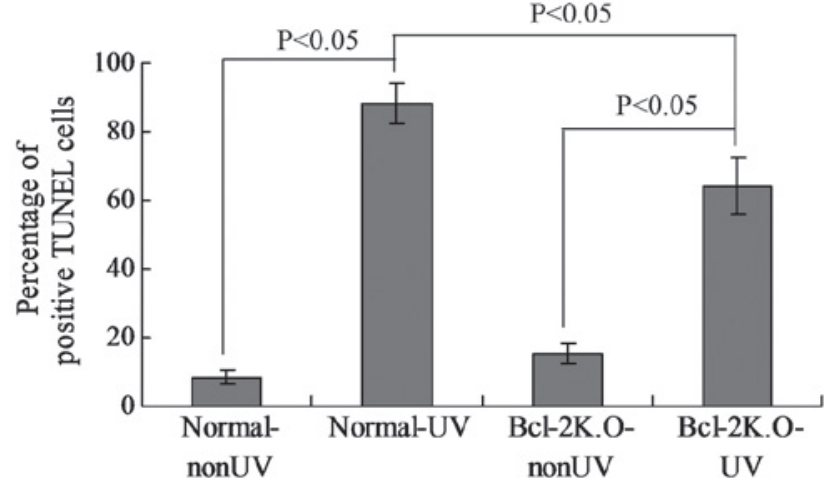

Figure 2. Apoptotic rates were analyzed in the crystalline lens of the various groups of samples using a terminal TUNEL assay. Sections were treated with terminal deoxynucleotidyl transferase and stained with fluorescein isothiocyanate-labelled deoxynucleotide triphosphate. (A) Image staining results (left upper quadrant, normal-nonUV group; right upper quadrant, normal-UV group; left lower quadrant, Bcl-2 K.O-nonUV group; right lower quadrant, Bcl-2 K.O-UV group) (magnification, x200) and (B) statistical analysis. UV, ultraviolet; K.O, knockout; TUNEL, terminal deoxynucleotidyl transferase dUTP nick-end labeling.

tosis in the tissue samples of the mouse crystalline lens of the various groups. The results of the present study indicated that large doses of UV radiation were able to induce a significant level of apoptosis in the epithelial cells of the crystalline lens (Fig. 2). The apoptotic rate was very low in the normal-nonUV group; however, a significant level of apoptosis was observed in the normal-UV and Bcl-2 K.O-UV groups (Fig. 2A; P<0.05). Furthermore, the apoptosis rate in the Bcl-2 K.O-nonUV group was not significant (Fig. 2B). Notably, the apoptotic rate in the normal-UV group was significantly higher, as compared with that of the $\mathrm{Bcl}-2$ K.O-UV group (Fig. 2B; P<0.05).

Bcl-2 protein can elevate the expression level of intracellular caspase-3. Caspase-3 is an important member in the family of cysteine-aspartic acid proteolytic enzymes. The enzyme is able to catalyze reactions of itself and other substrates, including caspase-7. The mRNA expression of caspase-3 was quantitatively determined using RT-qPCR analysis. The results indicated that the mRNA expression of caspase- 3 in the normal-UV group was significantly higher compared with the normal-nonUV group $(\mathrm{P}<0.05)$, while the mRNA expression in the Bcl-2 K.O-UV group was also significantly higher compared with the Bcl-2 K.O-nonUV group $(\mathrm{P}<0.05)$. In addition, the mRNA expression of caspase- 3 in the normal-UV group was significantly higher compared with that in the $\mathrm{Bcl}-2 \mathrm{~K} . \mathrm{O}-\mathrm{UV}$ group $(\mathrm{P}<0.05)$, as demonstrated in Fig. 3.

Bcl-2 protein can elevate the activity of caspase-3 protease. Further analysis of caspase-3 activity indicated that UV radiation significantly increased the activity of the proteolytic enzyme. In addition, caspase-3 activity levels were significantly higher in the normal-UV group when compared with the Bcl-2 K.O-UV group $(\mathrm{P}<0.05)$, under the same dose of UV radiation (Fig. 4). Therefore, following the determination of mRNA expression and the proteinase activity of caspase-3, the results indicated that the expression of the $\mathrm{Bcl}-2$ protein aggravates UV-induced apoptosis in the crystalline lens.

\section{Discussion}

Previous studies have demonstrated that $\mathrm{Bcl}-2$ mRNA transcription is present in the mouse crystalline lens during embryonic development, and the expression of Bcl-2 occurs following the appearance of ganglion cells in the retina $(13,14)$. The expression of $\mathrm{Bcl}-2$ has also been demonstrated in primary lens fibers. Expression has not been detected in the epithelial cells at the center of the anterior envelope of the crystalline lens; however, transcription has been shown to initiate when these cells move to the equator $(15,16)$. The specificity of tissue localization indicates that $\mathrm{Bcl}-2$ is transcribed and expressed 


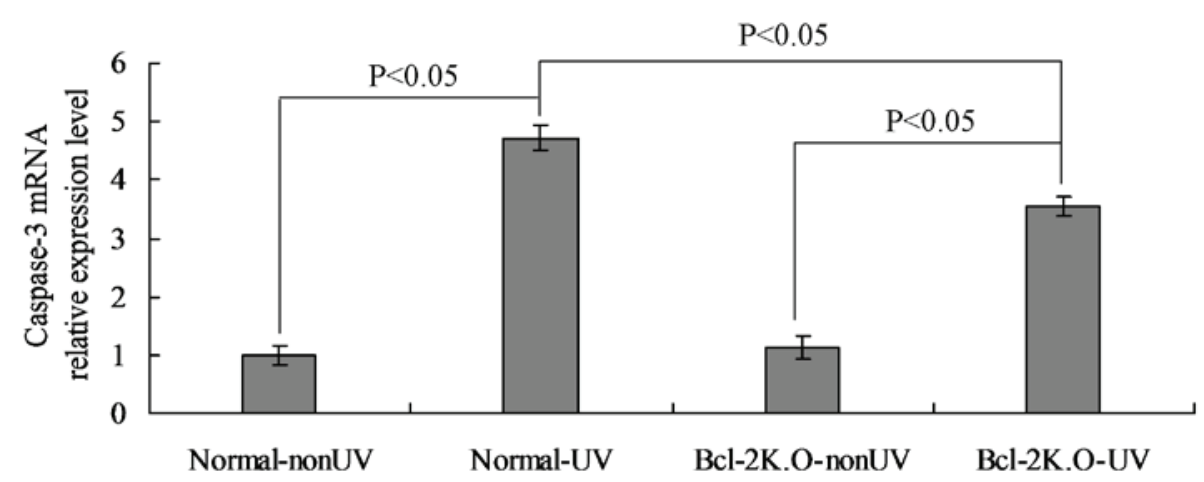

Figure 3. Reverse transcription-quantitative polymerase chain reaction was used to determine the relative mRNA expression levels of caspase-3 in the various groups. $\mathrm{P}<0.05$ vs. the illustrated groups. UV, ultraviolet; K.O, knockout.

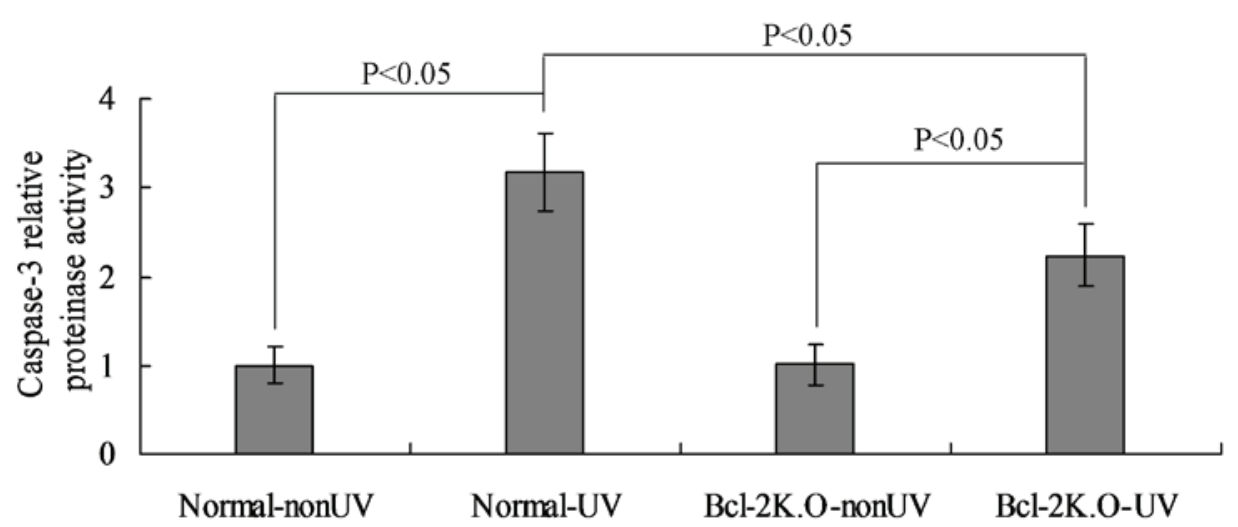

Figure 4. Determination of caspase-3 proteinase activity in the various groups. $\mathrm{P}<0.05$ vs. the illustrated groups. UV, ultraviolet; K.O, knockout.

in the fibrocytes of the epithelial and crystalline lens that are present in the equator area. With a relatively active growth, Bcl-2 may participate in a number of physiological processes associated with development; for example, $\mathrm{Bcl}-2$ may promote the expression of important structural proteins in cells, or have a role in the structural formation and functional maturation of the crystalline lens $(17,18)$. In the present study, the transcriptional expression of Bcl-2 mRNA was detected in the crystalline lens of adult mice, indicating that $\mathrm{Bcl}-2$ may participate in certain physiological processes of mature crystalline lens tissues.

The results of the present study demonstrated that Bcl-2 is capable of elevating the level of UV-induced apoptosis in the crystalline lens; thus, the protein may play a certain promoting role during apoptosis. In addition, the same result has been confirmed at a cellular level in previous studies $(19,20)$. The promoting role may be due to the action of the $\mathrm{Bcl}-2$ protein on a certain factor in the known apoptosis pathway. Bcl-2 may also affect the mechanism underlying apoptosis signaling transduction, which has yet to be fully elucidated (21). The internal and external pathways of apoptosis can activate the precursor of caspase-3, pro-caspase-3, to reveal the activity of the proteolytic enzyme; therefore, damaging the cellular structure via apoptosis and subsequently forming a series of morphological changes in significance $(22,23)$.

Caspase- 3 is an important indicator of apoptosis, and the activity of the enzyme presents the apoptosis capacity in a numeric form in order to conduct quantitative research $(24,25)$.
In the present study, the significant increase in caspase- 3 activity confirmed the positive role of $\mathrm{Bcl}-2$ in apoptosis. In addition, the experimental results demonstrated that UV radiation does not cause any change in the expression levels of $\mathrm{Bcl}-2$, indicating that $\mathrm{Bcl}-2$ is the regulating object in DNA damage. Therefore, the effect of the changes in the intrinsic protein level of Bcl-2 on apoptosis can be excluded when comparing the difference in the apoptosis rate in the crystalline lens between the group of K.O mice and the group of normal control mice that had been subjected to the same UV radiation conditions.

UV light is a component in solar radiation. Individuals may be more or less exposed to UV radiation every day. In particular, for eyes receiving external light for imaging, a small-dose of UV radiation accompanies each healthy individual during their lifetime. Thus, how to offset the damage of UV radiation to human eyes is the premise for maintaining normal visual functions. Bcl-2 may increase the sensitivity of cells in the crystalline lens to UV radiation damage, allowing the abnormal cells to be identified as early as possible and eliminated by apoptosis to ensure that the functions of the crystalline lens are not interfered by these abnormal cells. In this respect, $\mathrm{Bcl}-2$ has a positive significance in maintaining cellular 'metabolism'. However, excessive UV radiation or the repeated accumulation of small doses of UV radiation may lead to extensive apoptosis and further induce the occurrence of cataracts $(26,27)$. Therefore, the role of Bcl-2 in apoptosis appears to be a contradictory with negative and positive aspects. 


\section{References}

1. Galichanin K, Svedlund J and Söderberg P: Kinetics of GADD45 $\alpha$, TP53 and CASP3 gene expression in the rat lens in vivo in response to exposure to double threshold dose of UV-B radiation. Exp Eye Res 97: 19-23, 2012.

2. Xin G, Du J, Wang YT and Liang TT: Effect of oxidative stress on heme oxygenase-1 expression in patients with gestational diabetes mellitus. Exp Ther Med 7: 478-482, 2014.

3. Hengerer FH, Conrad-Hengerer I, Buchner SE and Dick HB Evaluation of the Calhoun Vision UV Light Adjustable Lens implanted following cataract removal. J Refract Surg 26: 716-721, 2010.

4. Mesa R and Bassnett S: UV-B-induced DNA damage and repair in the mouse lens. Invest Ophthalmol Vis Sci 54: 6789-6797, 2013.

5. Deng ZC, Zhao P, Cao C, Sun SF, Zhao F, Lu CY and Ma HY: $\mathrm{C}$-reactive protein as a prognostic marker in chronic obstructive pulmonary disease. Exp Ther Med 7: 443-446, 2014.

6. Kubo E, Hasanova N, Tanaka Y, Fatma N, Takamura Y, Singh DP and Akagi Y: Protein expression profiling of lens epithelial cells from Prdx6-depleted mice and their vulnerability to UV radiation exposure. Am J Physiol Cell Physiol 298: C342-C354, 2010.

7. Kalay S, Oztekin O, Tezel G, Aldemir H, Sahin E, Köksoy S, Akçakus $\mathrm{M}$ and Oygur N: Role of immunoglobulin in neuronal apoptosis in a neonatal rat model of hypoxic ischemic brain injury. Exp Ther Med 7: 734-738, 2014.

8. Varma SD, Hegde KR and Kovtun S: UV-B-induced damage to the lens in vitro: Prevention by caffeine. J Ocul Pharmacol Ther 24: 439-444, 2008.

9. Heo J, Lee BR and Koh JW: Protective effects of epigallocatechin gallate after UV irradiation of cultured human lens epithelial cells. Korean J Ophthalmol 22: 183-186, 2008.

10. Avetisov SE, Polunin GS, Sheremet NL, Makarov IA Fedorov AA, Karpova OE, Muranov KO, Dizhevskaia AK, Soustov LV, Chelnokov EV, et al: Chaperon-like anticataract agents, the antiaggregants of lens crystallin. Communication 4. Study of the effect of a mixture of di- and tetrapeptides on a prolonged rat model of UV-induced cataract. Vestn Oftalmol 124: 12-16, 2008. (In Russian).

11. Kernt M, Neubauer AS, Liegl R, Eibl KH, Alge CS Lackerbauer CA, Ulbig MW and Kampik A: Cytoprotective effects of a blue light-filtering intraocular lens on human retinal pigment epithelium by reducing phototoxic effects on vascular endothelial growth factor-alpha, Bax, and Bcl-2 expression. J Cataract Refract Surg 35: 354-362, 2009.

12. Galichanin K, Löfgren S, Bergmanson $\mathrm{J}$ and Söderberg P: Evolution of damage in the lens after in vivo close to threshold exposure to UV-B radiation: Cytomorphological study of apoptosis. Exp Eye Res 91: 369-377, 2010.

13. Gao J, Zhao L, Wang Y, Teng Q, Liang L and Zhang J: Effect of limb ischemic preconditioning on myocardial apoptosis-related proteins in ischemia-reperfusion injury. Exp Ther Med 5: $1305-1309,2013$

14. Avetisov SE, Polunin GS, Sheremet NL, Muranov KO, Makarov IA, Fedorov AA, Karpova OE and Ostrovskiŭ MA: Search for chaperon-like anticataract agents, the antiaggregants of lens crystallin. Communication 3. Possibilities of a follow-up of caractogenesis processes on a prolonged rat model of UV-induced cataract. Vestn Oftalmol 124: 8-12, 2008. (In Russian).
15. Wu XH, Lu Y, Fang YW and Jiang YX: The polyamidoamine-mediated inhibition of bcl-2 by small hairpin RNA to induce apoptosis in human lens epithelial cells. Mol Vis 18: 74-80, 2012.

16. Giblin FJ, Lin LR, Simpanya MF, Leverenz VR and Fick CE: A Class I UV-blocking (senofilcon A) soft contact lens prevents UVA-induced yellow fluorescence and NADH loss in the rabbit lens nucleus in vivo. Exp Eye Res 102: 17-27, 2012.

17. Soustov LV, Chelnokov EV, Sapogova NV, Bitiurin NM, Nemov VV, Karpova OE, Sheremet NL, Polunin GS, Avetisov SE and Ostrovskiŭ MA: Like anticataract agents, the antiaggregants of lens crystallin. Communication 2. Study of the impact of chaperon-like (protective) activity of short-chain peptides on the rate of UV-induced aggregation of betaL-crystallins by eximer laser. Vestn Oftalmol 124: 6-8, 2008. (In Russian).

18. Jiang Q, Cao C, Zhou C, Song X, Healey S, Kouttab N, Chu W, Xu A, Bi Z and Wan Y: Quercetin attenuates UV- and $\mathrm{H}(2) \mathrm{O}(2)$-induced decrease of collagen type I in cultured human lens epithelial cells. J Ocul Pharmacol Ther 24: 164-174, 2008.

19. Wang $Z$ and Wang $Z$ : Effects of rapamycin on expression of Bcl-2 and Bax in human lens epithelial cells and cell cycle in rats. J Huazhong Univ Sci Technolog Med Sci 31: 555-559, 2011.

20. Mafia K, Gupta R, Kirk M, Wilson L, Srivastava OP and Barnes S: UV-A-induced structural and functional changes in human lens deamidated alphaB-crystallin. Mol Vis 14: 234-248, 2008.

21. Kernt M, Hirneiss C, Neubauer AS, Ulbig MW and Kampik A: Coenzyme Q10 prevents human lens epithelial cells from light-induced apoptotic cell death by reducing oxidative stress and stabilizing BAX / Bcl-2 ratio. Acta Ophthalmol 88: e78-e86, 2010.

22. Pajer V, Tiboldi A, Bae N, Li K, Kang SU, Hopp B, Kolozsvári L, Lubec $\mathrm{G}$ and Nógrádi A: The molecular background of the differential UV absorbance of the human lens in the 240-400 nm range. Photochem Photobiol 89: 856-863, 2013.

23. Basu S, Rajakaruna S, De Arcangelis A, Zhang L, Georges-Labouesse E and Menko AS: $\alpha 6$ integrin transactivates insulin-like growth factor receptor-1 (IGF-1R) to regulate caspase-3-mediated lens epithelial cell differentiation initiation. J Biol Chem 289: 3842-3855, 2014

24. Lee HE, Choi ES, Shin JA, Lee SO, Park KS, Cho NP and Cho SD: Fucoidan induces caspase-dependent apoptosis in MC3 human mucoepidermoid carcinoma cells. Exp Ther Med 7: 228-232, 2014

25. Basu S, Rajakaruna S and Menko AS: Insulin-like growth factor receptor- 1 and nuclear factor $\kappa \mathrm{B}$ are crucial survival signals that regulate caspase-3-mediated lens epithelial cell differentiation initiation. J Biol Chem 287: 8384-8397, 2012.

26. Wang TT and Xu GX: [Effect of cinobufagin on the expressions of bcl-2 mRNA and bax mRNA and the proliferation of lens epithelial cells]. Zhongguo Zhong Xi Yi Jie He Za Zhi 29: 915-917, 2009. (In Chinese).

27. Lou JS, Chen XE, Zhang Y, Gao ZW, Chen TP, Zhang GQ and $\mathrm{Ji} \mathrm{C}$ : Photoprotective and immunoregulatory capacity of ginsenoside $\mathrm{Rg} 1$ in chronic ultraviolet B-irradiated BALB/c mouse skin. Exp Ther Med 6: 1022-1028, 2013. 